\title{
A Simulation Study of GNSS-R Polarimetric Scattering from the Bare Soil Surface Based on the AIEM
}

\author{
Xuerui $W u \mathbb{i D}^{1,2,3}$ and Shuanggen Jin ${ }^{1,2,3}$ \\ ${ }^{1}$ Shanghai Astronomical Observatory, Chinese Academy of Sciences, Shanghai 200030, China \\ ${ }^{2}$ Key Laboratory of Space Navigation and Position Technology, Shanghai 200030, China \\ ${ }^{3}$ School of Remote Sensing and Geomatics Engineering, Nanjing University of Information Science and Technology, \\ Nanjing 210044, China \\ Correspondence should be addressed to Xuerui Wu; xrwu@shao.ac.cn
}

Received 29 November 2018; Revised 20 March 2019; Accepted 7 April 2019; Published 8 May 2019

Academic Editor: Anthony R. Lupo

Copyright (c) 2019 Xuerui Wu and Shuanggen Jin. This is an open access article distributed under the Creative Commons Attribution License, which permits unrestricted use, distribution, and reproduction in any medium, provided the original work is properly cited.

\begin{abstract}
In the past two decades, global navigation satellite system-reflectometry (GNSS-R) has emerged as a new remote sensing technique for soil moisture monitoring. Some experiments showed that the antenna of V polarization is more favorable to receive the reflected signals, and the interference pattern technique (IPT) was used for soil moisture and retrieval of other geophysical parameters. Meanwhile, the lower satellite elevation angles are most impacted by the multipath. However, electromagnetic theoretical properties are not clear for GNSS-R soil moisture retrieval. In this paper, the advanced integral equation model (AIEM) is employed using the wave-synthesis technique to simulate different polarimetric scatterings in the specular directions. Results show when the incident angles are larger than $70^{\circ}$, scattering at RR polarization (the transmitted signal is right-hand circular polarization (RHCP), while the received one is also RHCP) is larger than that at LR polarization (the transmitted signal is RHCP, while the received one is left-hand circular polarization (LHCP)), while scattering at LR polarization is larger than that at RR polarization for the other incident angles $\left(1^{\circ} \sim 70^{\circ}\right)$. There is an apparent dip for VV and VR scatterings due to the Brewster angle, which will result in the notch in the final receiving power, and this phenomenon can be used for soil moisture retrieval or vegetation corrections. The volumetric soil moisture (vms) effects on their scattering are also presented. The larger soil moisture will result in lower scattering at RR polarization, and this is very different from the scattering of the other polarizations. It is interesting to note that the surface correlation function only affects the amplitudes of the scattering coefficients at much less level, but it has no effects on the angular trends of RR and LR polarizations.
\end{abstract}

\section{Introduction}

Soil moisture is the link between surface water and groundwater, which plays an important role in terrestrial ecosystems and water cycle systems. As one of the determinants in the status of the terrestrial hydrosphere, soil moisture largely controls the evapotranspiration, water migration, and carbon cycle on the land surface. Soil moisture is also important for understanding and forecasting climate change, solving global water cycle, water resources management, and basin hydrological modeling. Meanwhile, it is also one of the necessary conditions for monitoring crop growth, droughts, and floods.
Soil moisture has strong spatial and temporal variation heterogeneity. The traditional ground and meteorological monitoring is difficult to meet the requirements. The development of satellite remote sensing technology provides a new method for monitoring. In the past two decades, GNSS$\mathrm{R}$ has emerged as a new promising remote sensing technique due to its unique advantages [1]. Its applications extend from mesoscale ocean remote sensing to the remote sensing of snow, ice, and land surface parameters [2-4]. As for the land scenario, the most important application is soil moisture monitoring. GNSS-R is an effective complement to existing radars and radiometers and can be a potential efficient technique for the validation of the L-band soil moisture 
satellites, such as SMOS (soil moisture satellite) [5] and SMAP (soil moisture active passive) mission [6]. However, as an emerging means of earth observation, there are many open problems that need to be solved, such as the polarization properties of the reflected signals.

Polarization is one of the most important properties of the electromagnetic waves. The polarization of a plane wave describes the shape and direction of the space trajectory formed by the end of the electric field vector as a function of time. However, different from the traditional linear polarizations of the microwave remote sensing, the transmitted polarization of GNSS (global navigation satellite system) is RHCP (right-hand circular polarization). When the GNSS signals reflect from the surface, their polarizations will change. Kavak et al. [7] pointed out that the reflected signals consisted of two parts, the circular polarization and the perpendicular polarization, which are dependent on the elevation angles. Several experiments have paid attention to the polarization study of GNSS reflected signals. In [7], the modified GPS receiver was used, and a horizontal-positioned antenna of LHCP (lefthand circular polarization) was used to collect the coherent energy of the direct signals and the reflected signals. In the SMEX 02/03 (soil moisture experiment 2002/2003) [8], the antennas of the global position system-reflectometry (GPS$\mathrm{R})$ receiver were different. An airborne GPS-R receiver, known as DMR (delay-Doppler maps receiver), was used with two antennas. The standard zenith RHCP antenna was used to collect the direct signal, and the nadir LHCP antenna was used for the collection of the surface-reflected signals. Meanwhile, the incident angles were from $15^{\circ}$ to $35^{\circ}[4]$.

As early as in 2000, Zavorotny and Voronovich have used the GO (geophysical optics) scattering model [2] to study the reflection polarizations of soil moisture [9]. They simulated the sensitivity of polarization ratios (LR/RR and $\mathrm{HR} / \mathrm{VR})$ to the soil moisture at larger incident angles $\left(60^{\circ}\right.$ and $70^{\circ}$ ) and concluded theoretically that the polarization ratios can be used for soil moisture measurement. Here, LR , $\mathrm{RR}, \mathrm{HR}$, and VR present the polarization of transmitted signal as RHCP, while the received polarizations are LHCP, RHCP, horizontal $(\mathrm{H})$, and vertical $(\mathrm{V})$, respectively. In the BAO-tower (Boulder Atmospheric Observatory-tower) experiment [10], a multipolarization receiver provided by the NASA Langley Research Center was used. In order to collect the surface reflected signals, a low-gain LHCP and four highgain antennas ( $\mathrm{V}$ and $\mathrm{H}$ polarizations, LHCP, and RHCP) were used. The incidence angle was $35^{\circ}$, and the incident azimuthal angle was $245^{\circ}$. However, the BAO-tower experiment did not support their original theoretical hypothesis [9].

At present, the interference pattern technique (IPT) was used for soil moisture and vegetation retrieval [11]. An antenna of $\mathrm{V}$ polarization was employed since the angular information would be masked due to Brewster's angle if an antenna of LHCP was used [12]. LEiMON (Land Monitoring with Navigation Signals) was a long-term GNSS-R experimental campaign, which was funded by the European Space Agency (ESA). The experiment was conducted in 2009 using a SAM sensor, antenna of RHCP was used to collect the direct signals and antenna of RHCP or LHCP was used to collect the reflected signals. The elevation angles were from $40^{\circ}$ to $80^{\circ}$. However, their theoretical and experimental results indicated that the reflectivity of both RR and LR was increasing with the increase of the incidence angle $\left(0 \sim 80^{\circ}\right)$, which was very different from the commonly used Fresnel theory of circular polarization in the GNSS-R study [2, 4]. Different from the specially designed GNSS-R receiver, the multipath information of the geodetic-quality GPS receivers could be used to remotely sense the soil moisture in the top $5 \mathrm{~cm}$ of soil [13-15]. The in situ measurements showed that the elevation angels with $5^{\circ} \sim 30^{\circ}$ were most impacted by the multipath [13-15].

In general, most of the GNSS-R soil moisture monitoring is based on the experimental study $[4,8,10,11,13-15]$. The simple combination of geometry is commonly used to proceed from linear polarization to circular polarization [2, 4, 13-15]. RR polarization reflection increases with the incidence angle increase, while LR reflection decreases. The trend is very different from the LEiMON experimental report. It should be highlighted that only the amplitude changes are considered [2, 4, 13-15], while phase differences are not considered as calculations from linear to circular [16]. However, with the development of GNSS-R, more attention should be paid to theoretical models since they can assist in the data interpretation, sensitivity analysis, experiment design, and others [17]. In this paper, we focus on the polarization (especially circular polarization) dependence of bare soil scattering using an AIEM $[18,19]$; wave-synthesis technique is used for arbitrary polarizations calculation in Section 2. Reflectivity of XR polarization and linear polarizations versus incident angles is presented in Section 3; XR polarization means that the transmitted signal is RHCP and any polarization that is circularly polarized (RHCP and LHCP) or linearly polarized ( $\mathrm{V}$, vertical or $\mathrm{H}$, horizontal) is received. From the Mueller matrix, the effects of volumetric soil moisture and surface roughness for different polarizations are evaluated in Section 4. Finally, a conclusion is given in Section 5.

\section{AIEM}

To overcome the ionospheric effects, the transmitted signals are RHCP, while the polarizations of the transmitted signals changed after reflected from the soil surface. As shown in Section 1, since the polarizations of the reflected signals are different, the linear (vertical and horizontal) and circular (RHCP and LHCP) antennas are used to receive the reflected signals. Therefore, different combinations of polarizations need further study in theory. In this section, the commonly used random rough-surface bistatic scattering model of AIEM has been employed. By using the method of wavesynthesis technique, the model can calculate the bistatic scattering coefficient of arbitrary polarization combination (both linear and circular). In this way, the developed bistatic scattering model can be used to study the full polarization characteristics of soil moisture. 
The traditional electromagnetic scattering models from random rough surfaces [17] are the Kirchhoff model (KA) and the small perturbation model (SPM). The former is suitable for surfaces with small curvatures or high frequency limit, while the latter is applicable to slightly rough surfaces or low frequency. The popular integral equation model (IEM) is developed to bridge the gap between the SPM and KA. However, its several assumptions limit the accuracy, especially for bistatic scattering. The subsequently developed AIEM is an improvement of the IEM $[18,20]$. Here, we focus on the polarization study using the developed surface scattering model for GNSS-R applications. It is pointed out that the received power is mainly coming from the first
Fresnel zone [21], and therefore, only the coherent scattering is considered in our scattering properties study.

The bistatic scattering coefficient is related to the modified Mueller matrix asfollows [17]:

$$
\sigma_{q p}^{\circ}=\frac{4 \pi}{A} \mathbf{M}, q, p=h, v
$$

where $\sigma_{q p}^{\circ}$ is the bistatic scattering coefficient, subscripts $q$ and $p$ are the polarizations of the received and transmitted signals, respectively, $A$ is the illuminated area, and the modified Mueller matrix $\mathbf{M}$ can be written in terms of the elements of the scattering matrix $S$ :

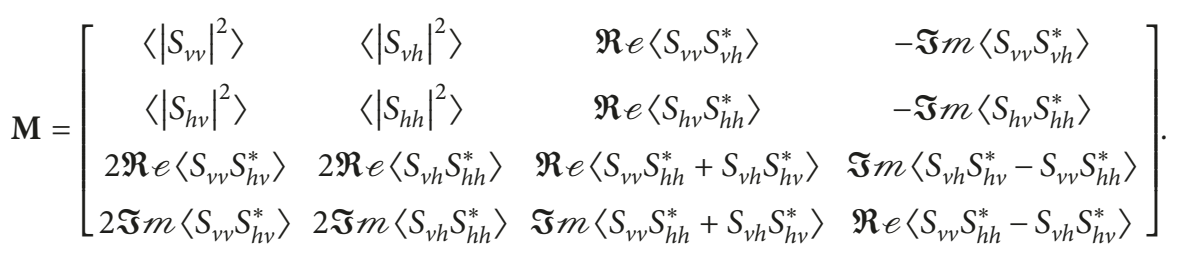

For more information of the AIEM model, see reference [20].

According to wave-synthesis technique [22], the scattering coefficient for a general transmitted and received antenna polarization can be written as

$$
\sigma_{q p}^{\circ}\left(\psi_{q}, \tau_{q} ; \psi_{q}, \tau_{q}\right)=\frac{4 \pi}{A}\left(\mathbf{I}_{q} \cdot \mathbf{Q} \cdot \mathbf{M} \cdot \mathbf{I}_{p}\right)
$$

where subscripts $q$ and $p$ are the polarizations of the received and transmitted signals, respectively, $\mathbf{I}$ is the normalized Stokes vectors with unit amplitude, and $\mathbf{Q}$ is a rotating matrix. The normalized Stokes vectors can be expressed in terms of the ellipticity angle $\chi$ and orientation angle $\psi$.

$$
\begin{gathered}
\mathbf{I}_{q}=\left[\begin{array}{c}
0.5\left(1+\cos 2 \tau_{q} \cos 2 \psi_{q}\right) \\
0.5\left(1-\cos 2 \tau_{q} \cos 2 \psi_{q}\right) \\
\cos 2 \tau_{q} \sin 2 \psi_{q} \\
\sin 2 \tau_{q}
\end{array}\right], \\
\mathbf{I}_{p}=\left[\begin{array}{c}
0.5\left(1+\cos 2 \tau_{p} \cos 2 \psi_{p}\right) \\
0.5\left(1-\cos 2 \tau_{p} \cos 2 \psi_{p}\right) \\
\cos 2 \tau_{p} \sin 2 \psi_{p} \\
\sin 2 \tau_{p}
\end{array}\right] .
\end{gathered}
$$

The first element of I describes the intensity of the electromagnetic wave in the horizontal direction and the vertical direction, the second one is the intensity difference between the horizontal and vertical directions of the wave vector, the third one is the linear polarization intensity at the azimuth angle of $\pm 45^{\circ}$ in the polarization ellipse, and the fourth one indicates the degree of circular polarization. In this way, the phase information in scattering matrix $S$ can be calculated through $\mathbf{I}$.
For like polarization, $\tau_{q}=\tau_{p}$ and $\psi_{q}=\psi_{p}+\pi$, and for a cross polarization, $\psi_{q}=\psi_{p}-\pi / 2$ and $\tau_{q}=-\tau_{p}$. Any polarizations of transmitted and received signals can be obtained by setting the ellipticity $\chi$ and orientation angle $\psi$, accordingly. For GNSS-R, it is necessary to study the scattering characteristics of $\sigma_{\mathrm{XR}}^{\circ}$. Subscript XR polarization means that the transmitted signal is RHCP and any polarization that is circularly polarized (RHCP and LHCP) or linearly polarized (V, vertical or $\mathrm{H}$, horizontal) is received.

\section{Reflectivity of Different Polarizations versus Incident Angles}

When a plane wave is transmitted through a plane boundary among the nondestructive media, there is an angle at which the vertical polarization broadcasts a full projection, which becomes the Brewster angle. The IPT method expressed in Section 1 uses the position and number information of the notches in the direct signal, and the reflected signals coherent waveform to perform the geophysical parameter retrieval. In fact, the notch in the waveform is the result of the Brewster angle. Due to its particularity, Brewster's angle is a phenomenon that needs attention as for GNSS-R remote sensing inversion. Table 1 presents the corresponding dielectric constants and the Brewster angles for different soil moistures. The vms is a way of expressing soil moisture, that is, the percentage of soil moisture volume. This kind of representation can reflect the degree of water filling of soil voids and can calculate the three ratios of soil solid, liquid, and gas. Figure 1 shows the Fresnel reflectivity of $r_{v}$ and $r_{h}$ polarization for different vms; here, $r$ means Fresnel reflectivity, while subscripts $v$ and $h$ mean the polarization state, $v$ stands for vertical polarization, and $h$ stands for horizontal. The corresponding Brewster angles in Table 1 are apparent at $\mathrm{V}$ polarization (left panel). As we can see if the 
TABLE 1: Dielectric constants and the Brewster angles for different vms.

\begin{tabular}{lcc}
\hline vms & Dielectric constant & Brewster angle \\
\hline 0.01 & $2.814-0.127 i$ & 59.2 \\
0.05 & $3.965-0.241 i$ & 63.33 \\
0.25 & $13.175-1.021 i$ & 74.597 \\
0.45 & $26.99-2.19 i$ & 79.1046 \\
0.60 & $40-3.3 i$ & 81.015 \\
\hline
\end{tabular}

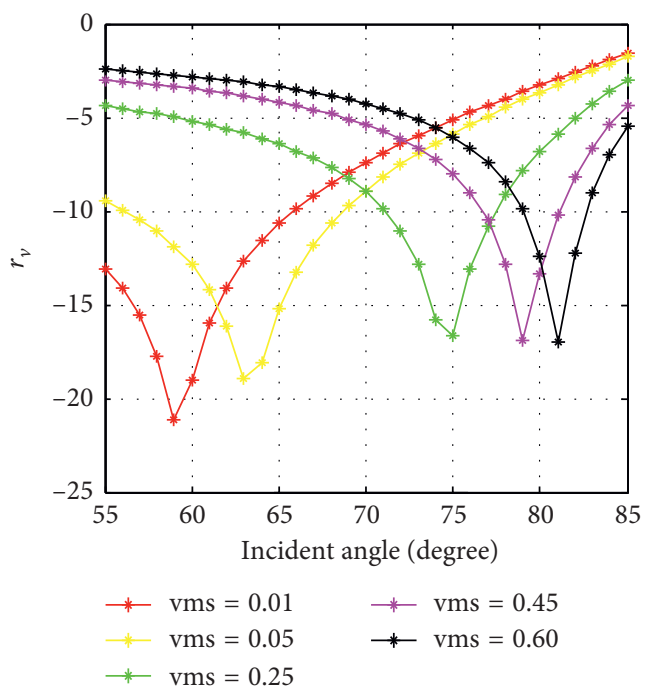

(a)

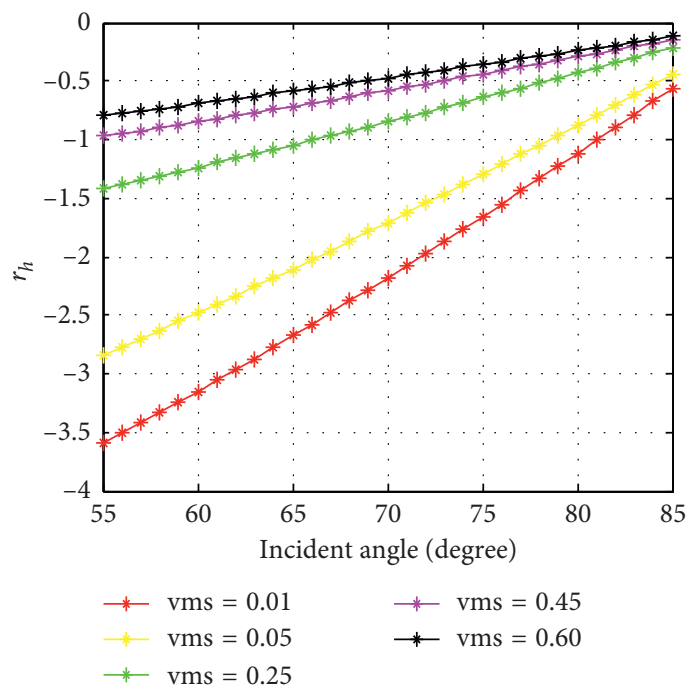

(b)

Figure 1: Fresnel reflectivity vs. incident angles: (a) $r_{v}$; (b) $r_{h}$.

vms is certain, the $r_{v}$ decreases with the incidence angle increase (before the Brewster angle) and the $r_{v}$ increases after the Brewster angle. As for the different vms, the $r_{v}$ is larger if the vms is larger (before $59^{\circ}$ ), but the $r_{v}$ is smaller if the vms is larger (after $82^{\circ}$ ). However, the $r_{h}$ is larger if the vms is larger.

The real parts of the $r_{v}$ and $r_{h}\left(\operatorname{Re}\left[r_{v}\right], \operatorname{Re}\left[r_{h}\right]\right)$ play an important role in the calculations of $\mathrm{XR}$ and linear polarization scattering coefficients. So, their changes with the incident angles are presented in Figure 2. Before hitting the Brewster angle, the $\operatorname{Re}\left[r_{v}\right]$ is positive, while it becomes negative if the incident angle is lower than the Brewster angle and the $\operatorname{Re}\left[r_{v}\right]$ increases with the incident angles increase. While $\operatorname{Re}\left[r_{h}\right]$ is negative for the whole angles with $\operatorname{Re}\left[r_{h}\right]$ decrease as vms increases.

Figure 3 is reproduced from [23], and it shows the scattering coefficients of linear polarization and XR polarization versus the incident angles. As we can see, for smaller incident angles $\left(1^{\circ}<\theta<70^{\circ}\right)$, the scattering of LR polarization is larger than that of $R R$ polarization $(\mathrm{RR}<\mathrm{LR})$. Here, $\mathrm{LR}$ and $\mathrm{RR}$ polarizations mean the transmitted signals are RHCP, while the received ones are LHCP and RHCP, respectively. However, for larger incident angles $\left(70^{\circ}<\theta<85^{\circ}\right)$, the trend is just opposite; that is to say, the scattering coefficients of $\mathrm{RR}$ polarization are larger than the ones of LR polarization. The scattering coefficients of RR polarization is larger than that of VR polarization for larger incident angles $\left(70^{\circ}<\theta<85^{\circ}\right)$; here, VR polarization means the transmitted signal is RHCP, while the received one is vertical polarization. The scattering coefficients of RR polarization increase as the incident angles increase $\left(1^{\circ}<\theta<55^{\circ}\right)$, but for the other incident angles, the scattering coefficient of RR polarization decreases. However, the scattering coefficients of LR polarization decrease as the incident angle increases. The trends of scattering coefficient of $\mathrm{VV}, \mathrm{VR}, \mathrm{HH}$, and $\mathrm{HR}$ versus incident angles are the same. Here, VR and $H R$ means the transmitted signals are RHCP, while the received signals are vertical and horizontal polarizations, respectively. $\mathrm{VV}$ and $\mathrm{HH}$ mean the same polarization of vertical and horizontal, respectively. The reason is that they are only related to $\mathbf{M}(1,1)$ and $\mathbf{M}(2,2)$, respectively (equations (2) and (5)). As the incident angles increase, the scattering coefficients of $\mathrm{HH}$ and $\mathrm{HR}$ polarizations decrease. Scattering coefficients of VV and VR polarizations have dips at about $70^{\circ}$ because of the Brewster angle defined in equation (8) [17]; the scattering coefficients decrease at first (before the Brewster angle) and then increase with the incidence angle increase (after the Brewster angle). 


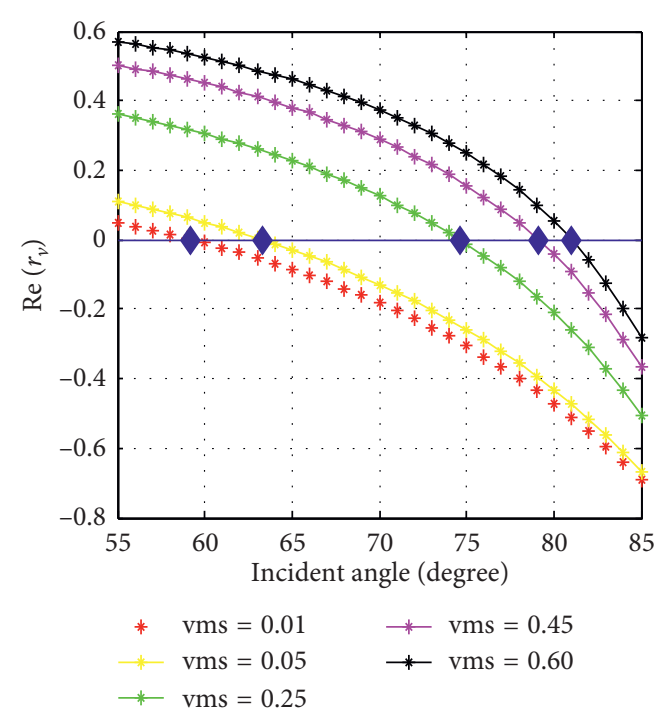

(a)

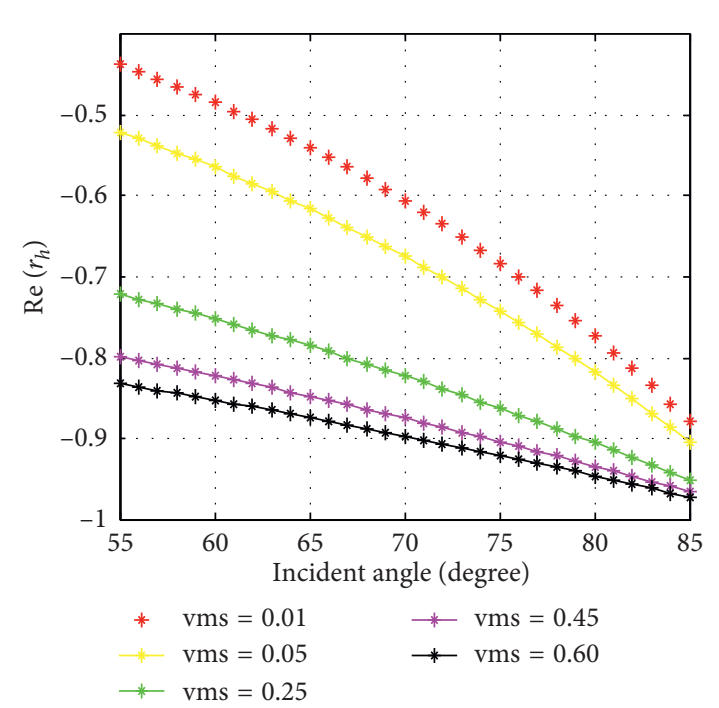

(b)

FIGURE 2: Real parts of the Fresnel reflectivity vs. the incident angles: (a) $\operatorname{Re}\left[r_{v}\right]$; (b) $\operatorname{Re}\left[r_{h}\right]$.

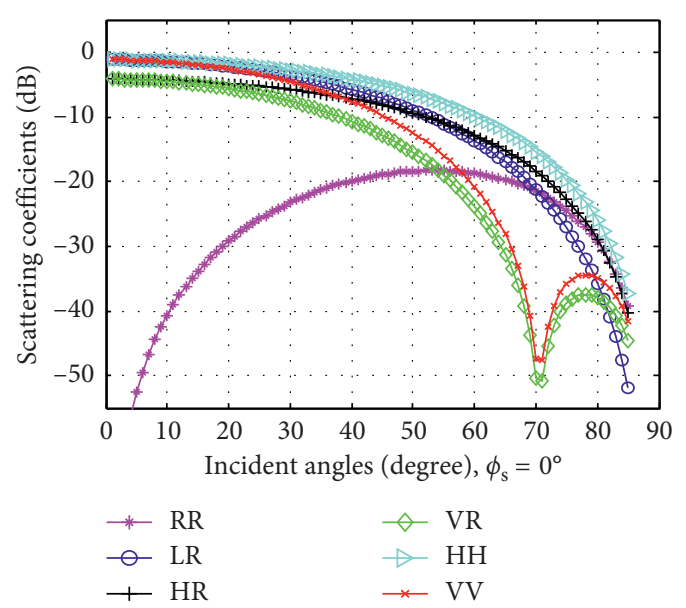

FIGURE 3: Scattering coefficients of linear polarization and $\mathrm{RX}$ polarization vs. incident angles $(\mathrm{vms}=0.15)$.

\section{Effects of Volumetric Soil Moisture and Surface Roughness on Polarizations}

4.1. Effects of Volumetric Soil Moisture on Polarizations. Figure 4 gives the linear and circular polarization scattering coefficients at different soil moisture (vms) versus the incident angles. The scattering trends of HR polarization are very similar to that of $\mathrm{HH}$ polarization, while VR polarization is very similar to VV polarization as shown in Figure 1. The dips at VV and VR polarization are related to the vms, and this is due to the Brewster angles (Table 1). If the vms is larger, the position of dip is at the position of larger incident angle. It should be noted that the scattering coefficients of RR polarization are larger if the vms is smaller. For the other polarizations (LR, HR, and $\mathrm{HH}$ ), larger vms corresponds to larger scattering coefficients. But, for $\mathrm{VV}$ and
VR polarizations, if the vms is larger, the scattering coefficients are larger if the incident angles are lower than the Brewster angles. While if the incident angles are larger than the Brewster angles, the lower vms corresponds to larger scattering coefficients.

4.2. Effects of Surface Roughness on Different Polarizations. Since the soil moisture affects the GNSS reflection signal, the geometric characteristics of the soil, that is, the surface roughness will also affect the GNSS reflection signal. Therefore, in the soil moisture inversion, how to remove the surface roughness is a difficult problem. The surface roughness can be represented by two parameters, the root mean square height $(s)$ and the surface correlation length $(l)$, which, respectively, define the surface roughness from the vertical and horizontal scales. Another way to describe the degree of random surface roughness is the surface autocorrelation function, which can be described by a Gaussian autocorrelation function or an exponential autocorrelation function.

In this section, surface roughness effects on the final scattering at RR and LR polarizations are presented. Figure 5 shows the roughness effects on different correlation functions. As we can see, if root square height $(s)$ is fixed, larger correlation length $(l)$ corresponds to larger scattering coefficients (both RR and LR); if $l$ is fixed, larger $s$ corresponds to larger scattering coefficients (both RR and LR), $k$ is the wave number.

The scattering of exponential correlation function is larger than the one of Gaussian function (both RR and LR) (Figure 6). It is interesting to note that unlike in backscattering direction, the surface correlation function only affects the amplitudes of the scattering coefficients, while the angular trend is at much less level. 


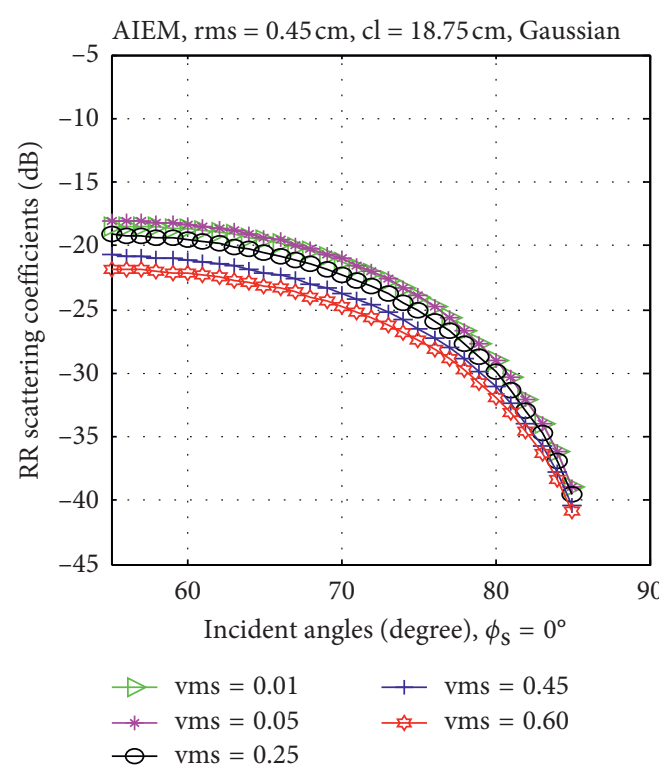

(a)

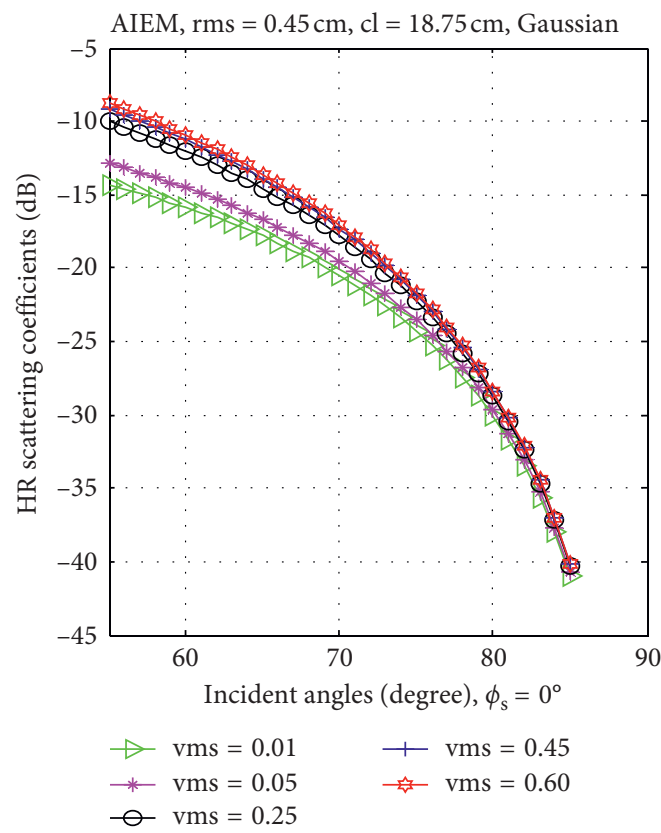

(c)

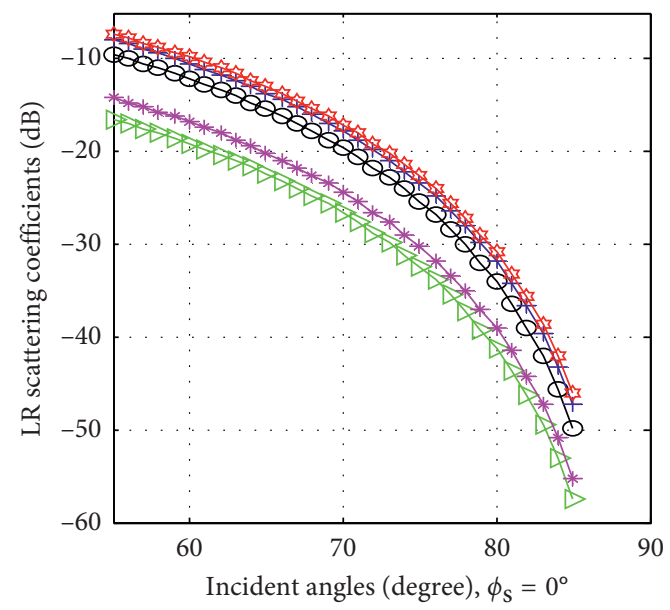

$$
\begin{array}{rlrl}
\rightarrow \mathrm{vms} & =0.01 \quad \text { 十 } \\
\rightarrow & \mathrm{vms}=0.05 & =0.45 \\
\text { vms } & =0.05 &
\end{array}
$$

(b)

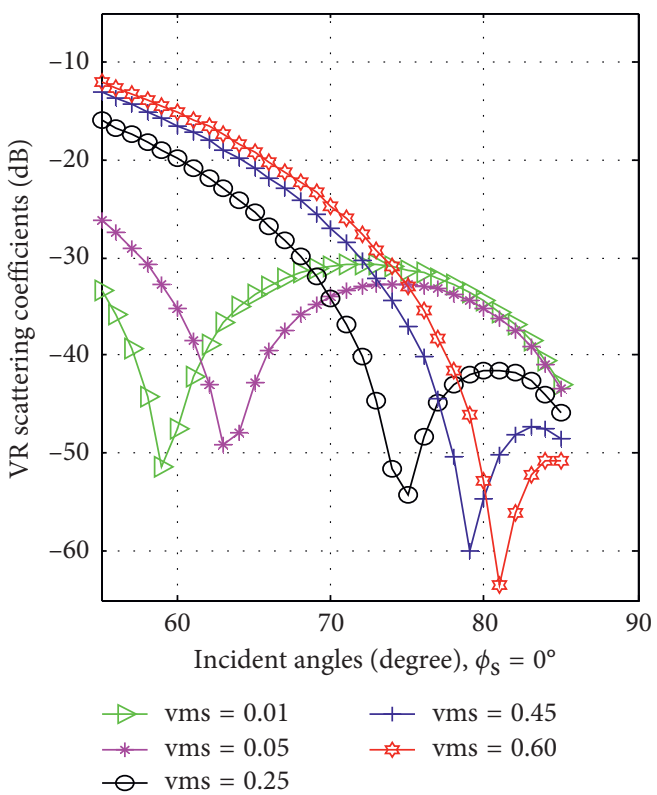

(d)

Figure 4: Continued. 


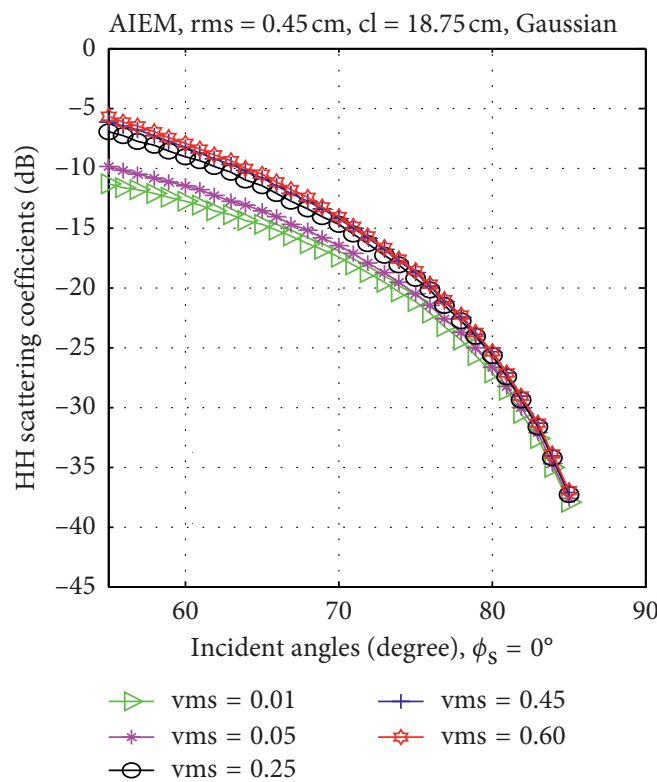

(e)

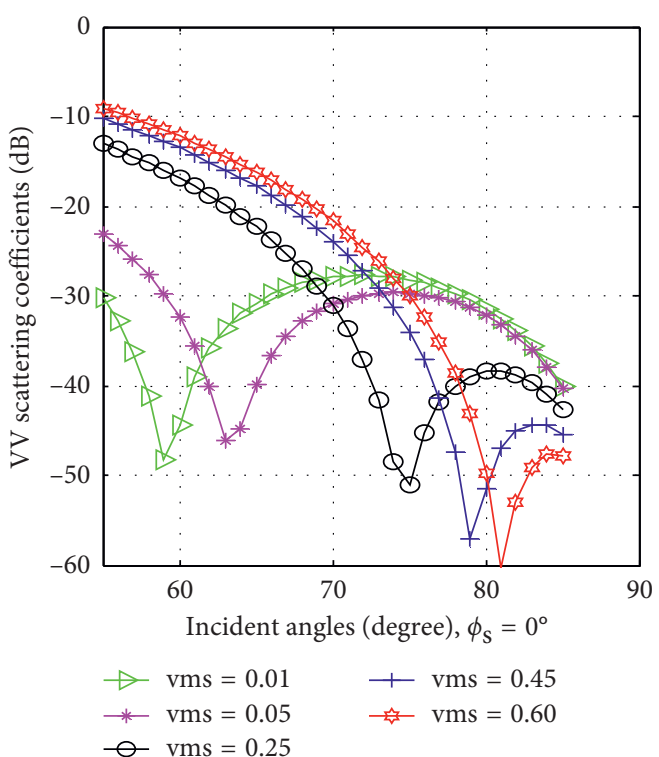

(f)

FIgURE 4: Scattering coefficients of different vms vs. the incident angles.

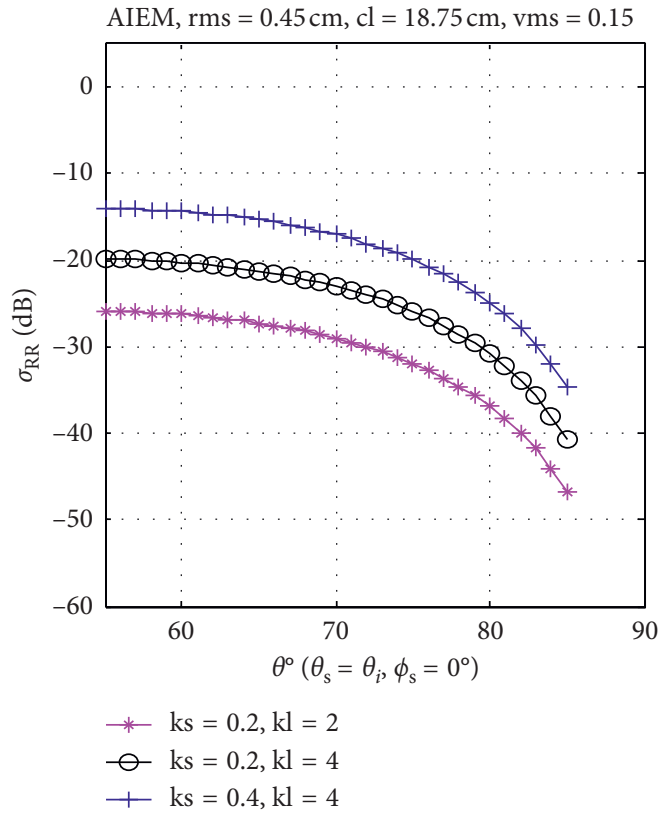

(a)

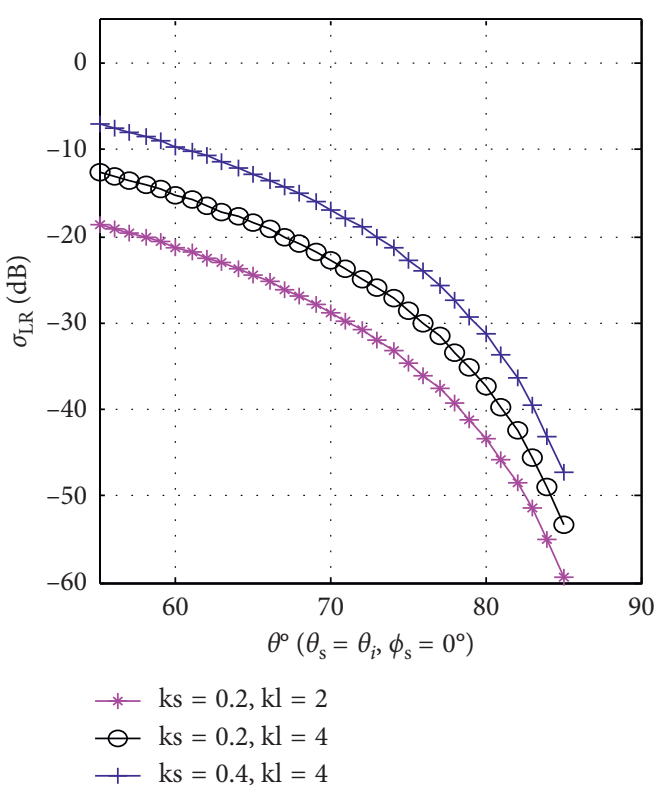

(b)

FIgURE 5: Surface roughness effects on the RR and LR scattering.

\section{Conclusions}

As for GNSS-R remote sensing, the polarization of transmitted signals is RHCP, which is commonly thought that the received power is coming from the first Fresnel zone. Here, the coherent scattering of different polarizations is simulated by the modified AIEM using the wave-synthesis technique. Results show that the scattering at RR polarization is lower than that at LR polarization as for incident angles lower than $70^{\circ}$; when the incident angles are between $70^{\circ}$ and $80^{\circ}$, the scattering at LR polarization is larger than that at RR polarization. As for larger incident angles, there are dips at VV and VR polarizations due to the Brewster angles, which are related to the soil moisture. Therefore, the antenna of $\mathrm{V}$ polarization is suggested to be used because the information of dips caused by the Brewster angle at different soil moistures is useful for soil moisture monitoring and the scattering dynamic ranges of VR polarization are larger than 


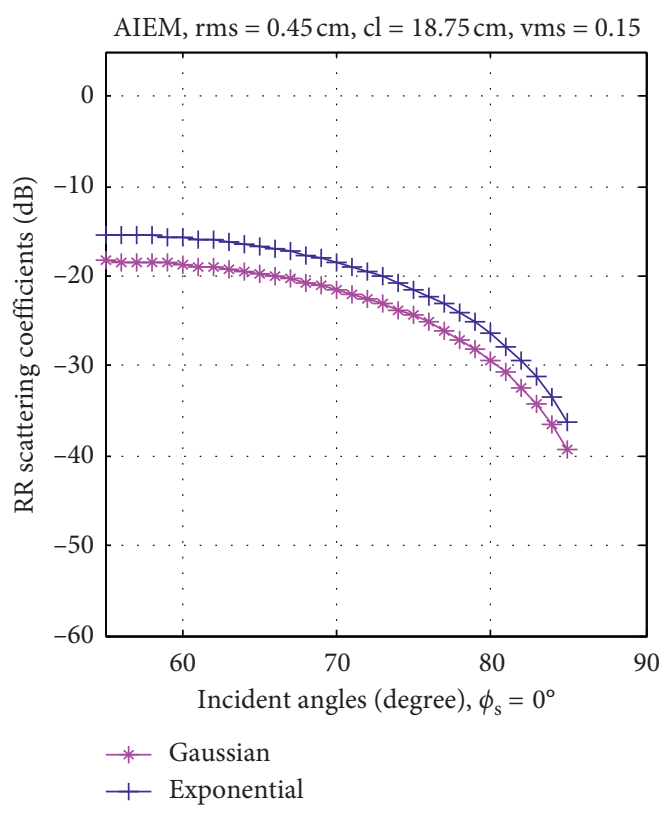

(a)

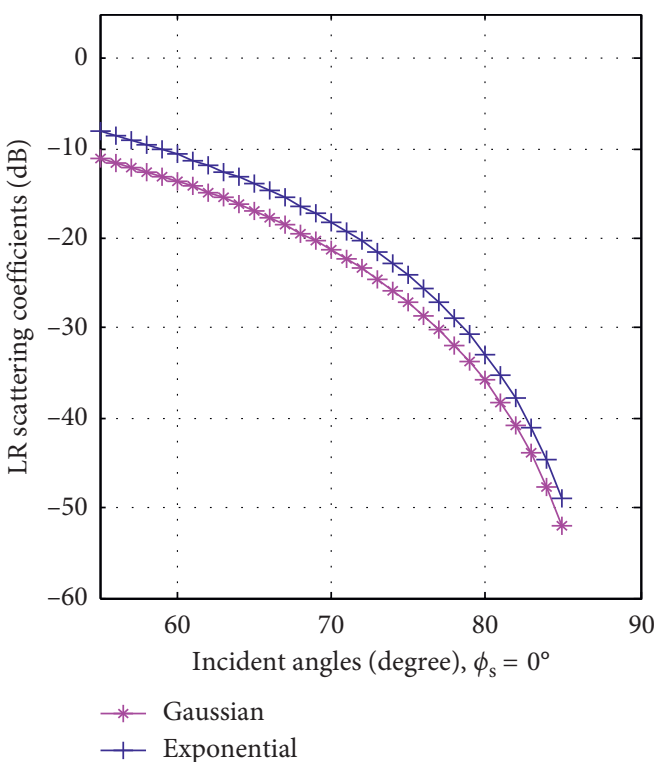

(b)

FIGURE 6: Correlation functions on the RR and LR scattering.

those of the other polarizations. Meanwhile, VR polarization will provide efficient information for vegetation corrections. It should be noted that larger soil moisture corresponds to lower scattering at RR polarization, while the trend is just the opposite as for the other XR (LR, HR, VR, HH, and VV) polarizations. Meanwhile, it is interesting to note that unlike in backscattering direction, the surface correlation function only affects the amplitudes of the scattering coefficients but it has no effects on the angular trend.

\section{Abbreviations}

AIEM:

DDM:

DMR:

GNSS:

GNSS-R:

GO:

GPS:

HR, VR, +45R, and $-45 \mathrm{R}$ polarizations:

IEM:

IPT:

KA:

LEiMON:

LHCP:

LR:

RHCP:
Advanced integral equation model Delay-Doppler maps Delay Doppler maps receiver GNSS-reflectometry Geophysical optics model Global positioning system The polarization of the transmitted signals is RHCP with the received ones $\mathrm{H}$ (horizontal), $\mathrm{V}$ (vertical), +45 $\left(+45^{\circ}\right.$ linear), and $-45\left(-45^{\circ}\right.$ linear $)$ polarizations, respectively Integral equation model Interference pattern technique Kirchhoff model Land Monitoring with Navigation Signals

Left-hand circular polarization The polarization of the transmitted signal is RHCP, while the received one is LHCP

Right-hand circular polarization Global navigation satellite System
RR:

SMAP:

SMEX:

SMIGOL:

SMOS:

SPM:

Z-V model:

\section{Data Availability}

The data used to support the findings of this study are available from the corresponding author upon request.

\section{Conflicts of Interest}

The authors declare that they have no conflicts of interest.

\section{Acknowledgments}

This research was supported by the National Natural Science Foundation of China (NSFC) Project (Grant no. 41501384) and the Strategic Priority Research Program Project of the Chinese Academy of Sciences (Grant no. XDA23040100).

\section{References}

[1] S. Jin and A. Komjathy, "GNSS reflectometry and remote sensing: new objectives and results," Advances in Space Research, vol. 46, no. 2, pp. 111-117, 2010. 
[2] V. U. Zavorotny and A. G. Voronovich, "Scattering of GPS signals from the ocean with wind remote sensing application," IEEE Transactions on Geoscience and Remote Sensing, vol. 38, no. 2, pp. 951-964, 2000.

[3] E. Cardellach, F. Fabra, A. Rius, S. Pettinato, and S. D’Addio, "Characterization of dry-snowsub-structure using GNSS reflected signals," Remote Sensing of Environment, vol. 124, pp. 122-134, 2012.

[4] D. Masters, P. Axelrad, and S. Katzberg, "Initial results of land-reflected GPS bistatic radar measurements in SMEX02," Remote Sensing of Environment, vol. 92, no. 4, pp. 507-520, 2004.

[5] M. Berger, A. Camps, J. Font et al., "Measuring ocean salinity with ESA's SMOS mission," ESA Bulletin Journal, vol. 111, no. 113, pp. 113-121, 2002.

[6] D. Entekhabi, E. Njoku, P. O'Neill et al., "The soil moisture active passive (SMAP) mission," Proceedings of the IEEE, vol. 98, no. 5, pp. 704-716, 2010.

[7] A. Kavak, G. Xu, and W. J. Vogel, "GPS multipath fade measurements to determine L-band ground reflectivity properties," in Proceedings of the 20th NASA Propagation Experimenters Meeting, Fairbanks, AK, USA, 1996.

[8] D. Masters, SMEX02 Airborne GPS Bistatic Radar Data, Iowa, University of Colorado, Colorado Center for Astrodynamics Research, Boulder, CO, USA, 2005.

[9] V. U. Zavorotny and A. G. Voronovich, "Bistatic GPS signal reflections at various polarizations from rough land surface with moisture content," in Proceedings of the IEEE International Geoscience Remote Sensing Symposium, vol. 7, pp. 2852-2854, Honolulu, HI, USA, July 2000.

[10] V. Zavorotny, D. Masters, A. Gasiewski et al., "Seasonal polarimetric measurements of soil moisture using towerbased gps bistatic radar," in Proceedings of the 2003 IEEE International Geoscience and Remote Sensing Symposium (IGARSS'03), vol. 2, pp. 781-783, Toulouse, France, July 2003.

[11] N. Rodriguez-Alvarez, A. Camps, M. Vall-llosera et al., "Land geophysical parameters retrieval using the interference pattern GNSS-R technique," IEEE Transactions on Geoscience and Remote Sensing, vol. 49, no. 1, pp. 71-84, 2011.

[12] N. Rodriguez-Alvarez, X. Bosch-Lluis, A. Camps et al., "Soil moisture retrieval using GNSS-R techniques: experimental results over a bare soil field," IEEE Transactions on Geoscience and Remote Sensing, vol. 47, no. 11, pp. 3616-3624, 2009.

[13] K. M. Larson, E. E. Small, E. D. Gutmann, A. L. Bilich, J. J. Braun, and V. U. Zavorotny, "Use of GPS receivers as a soil moisture network for water cycle studies," Geophysical Research Letters, vol. 35, no. 24, article L24405, 2008.

[14] K. M. Larson, J. J. Braun, E. E. Small, V. U. Zavorotny, E. D. Gutmann, and A. L. Bilich, "GPS multipath and its relation to near-surface soil moisture content," IEEE Journal of Selected Topics in Applied Earth Observations and Remote Sensing, vol. 3, no. 1, pp. 91-99, 2010.

[15] C. C. Chew, E. D. Small, K. M. Larson, and V. Zavorotny, "Effects of near-surface soil moisture on GPS SNR data: development of a retrieval algorithm for soil moisture," IEEE Transactions on Geoscience and Remote Sensing, vol. 52, no. 1, pp. 537-543, 2013.

[16] G. Ruck, D. Barrick, W. Stuart et al., Radar Cross Section Handbook, Plenum, Vol. 2, New York, NY, USA, 1970.

[17] A. K. Fung, Microwave Scattering and Emission Models and Their Applications, Artech House, Norwood, MA, USA, 1994.

[18] K. S. Chen, T.-D. Wu, L. Tsang, Q. Li, S. Shi, and A. K. Fung, "Emission of rough surfaces calculated by the integral equation method with comparison to three-dimensional moment method simulations," IEEE Transactions on Geoscience and Remote Sensing, vol. 41, no. 1, pp. 90-101, 2003.

[19] X. Wu and S. Jin, "Characteristics of GNSS-R bare soil based on advanced integral equation model (AIEM) and interference patterns," in Proceedings of the General Assembly \& Scientific Symposium, IEEE, Beijing, China, August 2014.

[20] T. D. Wu, K. S. Chen, J. C. Shi, H. W. Lee, and A. K. Fung, "A study of AIEM model for bistatic scattering from randomly surfaces," IEEE Transactions on Geoscience and Remote Sensing, vol. 46, no. 9, pp. 2584-2598, 2008.

[21] P. Beckmann and A. Spizzichino, The Scattering of Electromagnetic Waves from Rough Surfaces, Artech House, Norwood, MA, USA, 1963.

[22] F. T. Ulaby and C. Elachi, Radar Polarimetry for Geoscience Applications, Artech House Publishers, Norwood, MA, USA, 1990.

[23] X. Wu and S. Jin, Sensing Bare Soil and Vegetation Using GNSS-R-Theoretical Modeling. Satellite Positioning: Methods, Models and Applications, InTech-Publisher, Rijeka, Croatia, 2015. 

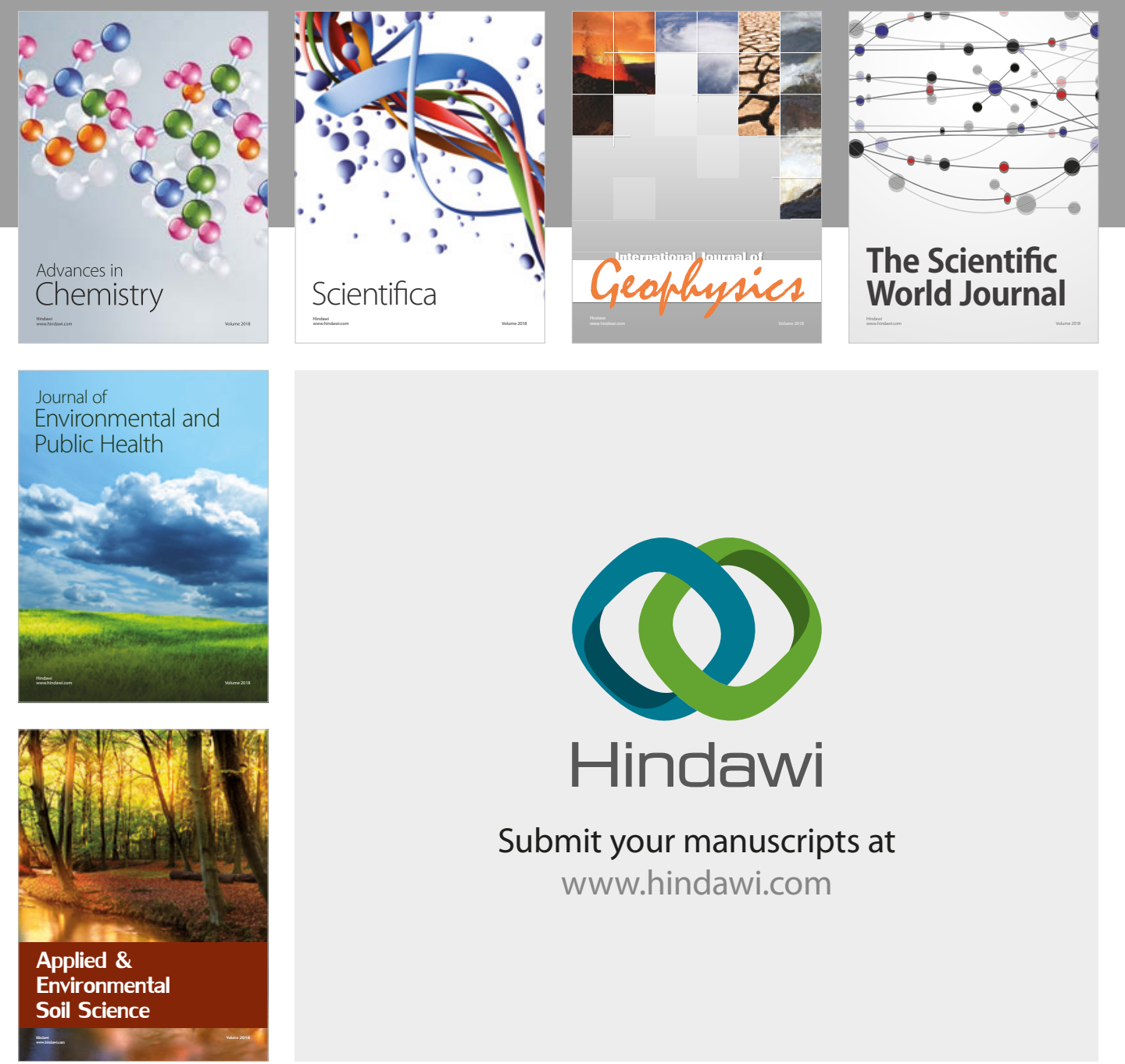

The Scientific

\section{World Journal}
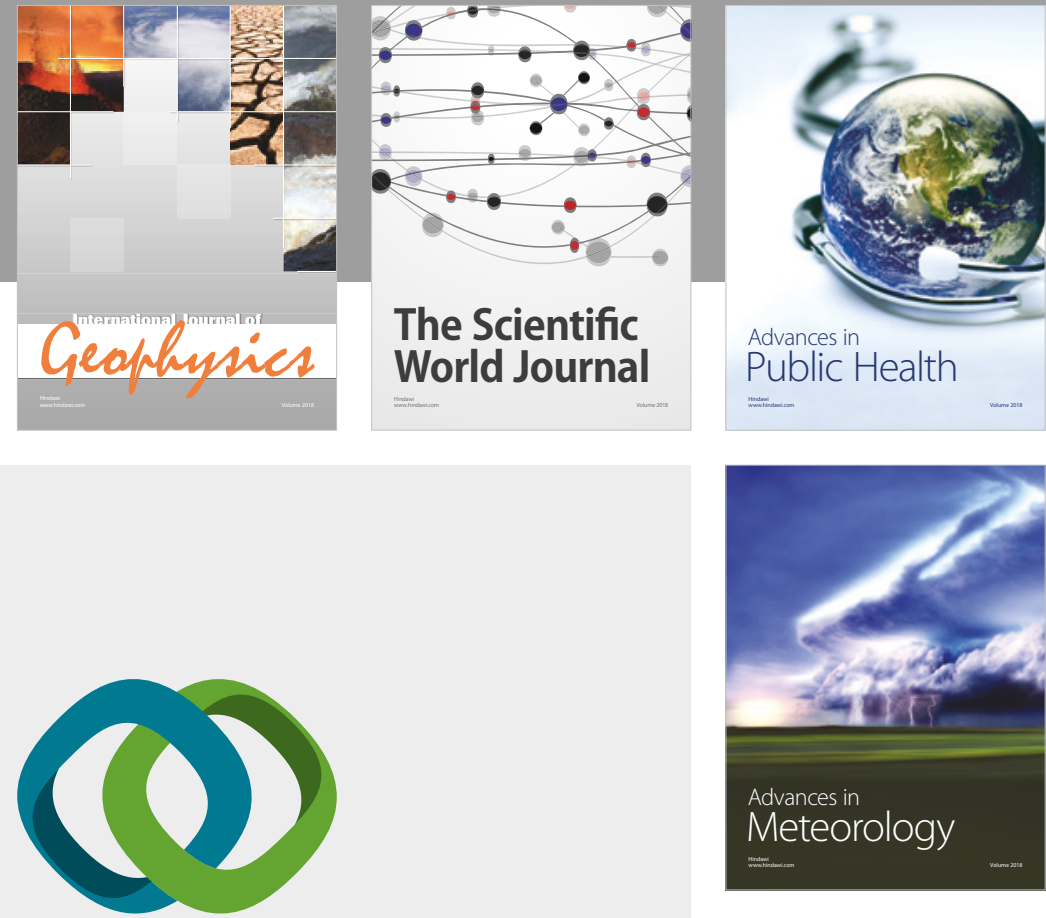

Advan

Public Health

\section{Hindawi}

Submit your manuscripts at

www.hindawi.com
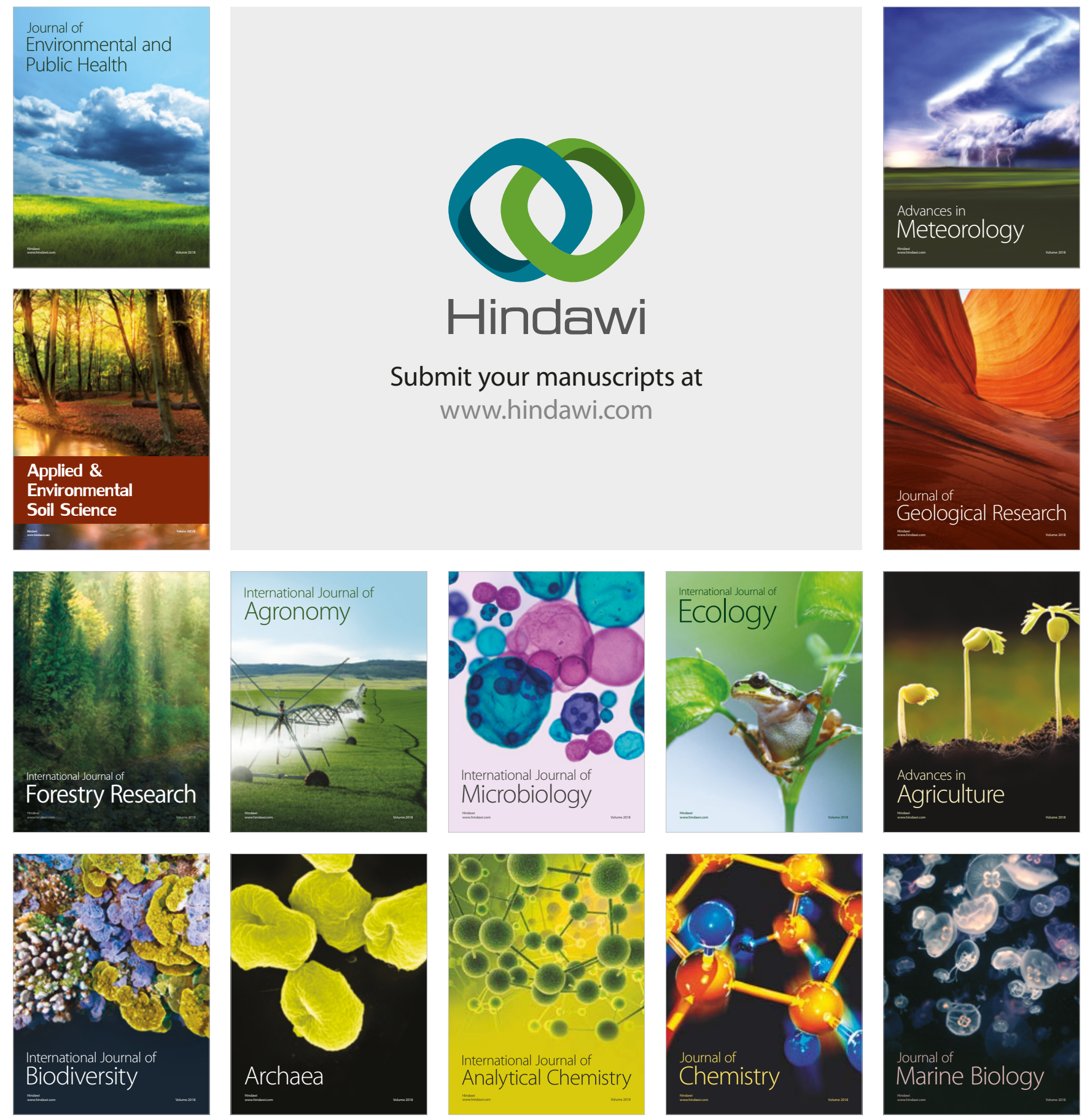